\begin{tabular}{|c|l|}
\hline Title & A local damage model for anomal ous high toughness of double network gels \\
\hline Author(s) & Tanaka, Y. \\
\hline Citation & $\begin{array}{l}\text { Europhysics Letters, 78(5), 56005 } \\
\text { https://doi.org/10.1209/0295-5075/78/56005 }\end{array}$ \\
\hline Issue Date & 2007 \\
\hline Doc URL & http://hdl.handle.net/2115/28252 \\
\hline Type & article \\
\hline File Information & EPL78-5.pdf \\
\hline
\end{tabular}

Instructions for use 


\title{
A local damage model for anomalous high toughness of double-network gels
}

\author{
Y. TANAKA \\ Creative Research Initiative Sousei, Hokkaido University - Sapporo 001-0021, Japan
}

received 19 January 2007; accepted in final form 25 April 2007

published online 22 May 2007

PACS 61.41.+e - Polymers, elastomers, and plastics

PACS 62.20.Mk - Fatigue, brittleness, fracture, and cracks

PACS 81.05.Qk - Reinforced polymers and polymer-based composites

\begin{abstract}
We present a phenomenological model for anomalously high fracture energy of doublenetwork (DN) gels, which consist of a substantially cross-linked polyelectrolyte gel (first network) and of a quite poorly cross-linked neutral polymer (second network) penetrating into the first network (Gong J. P., Katsuyama Y., Kurokawa T. and Osada Y., Adv. Mater., 15 (2003) 1155). The model assumes that the material locally softens around crack tip due to damage of the first network, and then the crack extends within the softened zone. An order estimation indicates that energy dissipation by the softening greatly exceeds the "bare fracture energy" of the softened material, and that the effective fracture energy can reach the order of $100 \mathrm{~J} / \mathrm{m}^{2}$. This is consistent with the experimental value $\sim 400 \mathrm{~J} / \mathrm{m}^{2}$.
\end{abstract}

Copyright (C) EPLA, 2007

Polymer scientists had though that succulent gels are very easy to break because of their large water contents. This stereotype thinking has been broken by the development of double-network (DN) gels [1], a kind of IPN (interpenetrating polymer network) consisting of a strong polyelectrolyte gel (first network) and of neutral long polymer chains (second network). For a DN gel synthesized at an adequate composition, the fracture energy $G$ reaches around $400 \mathrm{~J} / \mathrm{m}^{2}[2]$; this value, despite about $85 \mathrm{wt} \%$ water content of the gel, exceeds the typical fracture energy of rubbers (without filler) at low crack velocities, $\sim 100 \mathrm{~J} / \mathrm{m}^{2}[3]$. In addition, DN gels remind us of the high toughness of gel-like animal tissues, such as cartilage and tendon, which are hybrids of rigid and flexible biopolymers. DN gels have provoked a lot of interest from fundamental and applied points of view, and there have been several investigations on the structure and mechanical properties of the gels $[1,2,4-6]$. However, the understanding of the toughening mechanism remains at the level of speculations at present.

The structural features of the tough DN gels are as follows. F1) The material combination is: the first network of poly(2-acrylamido-2-methylpropanesulfonic acid), PAMPS, and the second network of polyacrylamide, PAAm. The former is very rigid, and the latter flexible. F2) PAMPS is substantially cross-linked, while PAAm chains are strongly entangled but not (or quite poorly) cross-linked. F3) The molar concentration of PAMPS, approximately $0.1 \mathrm{M}$, is fairly lower than that of PAAm, approximately $2 \mathrm{M}$. These features indicate that the first and second networks have quite brittle and ductile natures, respectively. On the other hand, mechanical characterizations on the DN gels have revealed that F4) despite the minority of PAMPS, the elastic modulus of the DN gels is dominated by the first PAMPS network, that is Young's modulus of the DN gels is almost the same as the corresponding bare PAMPS gels (the PAMPS gels used as the first network). This is because the elasticity of PAMPS gels (and DN gels) mainly comes from the activity of dissociated counter ions. In addition, the following experimental finding is quite crucial in discussions on the anomalous high fracture energy $G$. F5) $G$ hardly depends on crack velocity $V$ : for the increase of $V$ over four orders (from $10^{-6}$ to $10^{-2} \mathrm{~m} / \mathrm{s}$ ), $G$ increases up to only three times [2].

We emphasise that the high mechanical strength of the DN gel cannot be explained by the well-known mechanisms to enhance the fracture energy $G$ of the soft-polymer systems, i.e., chain stretching [7] and sliding [8] in vicinity of crack tip, and bulk vicoelasticity [9]. For example, if the fracture energy is dominated by the chain stretching effect of PAAm, $G$ should be around $10 \mathrm{~J} / \mathrm{m}^{2}$ for the PAAm concentration of $2 \mathrm{M}$, as estimated in [2]. This value is 1-2 orders less than the $G$ of the tough DN gels [2]. The chain 


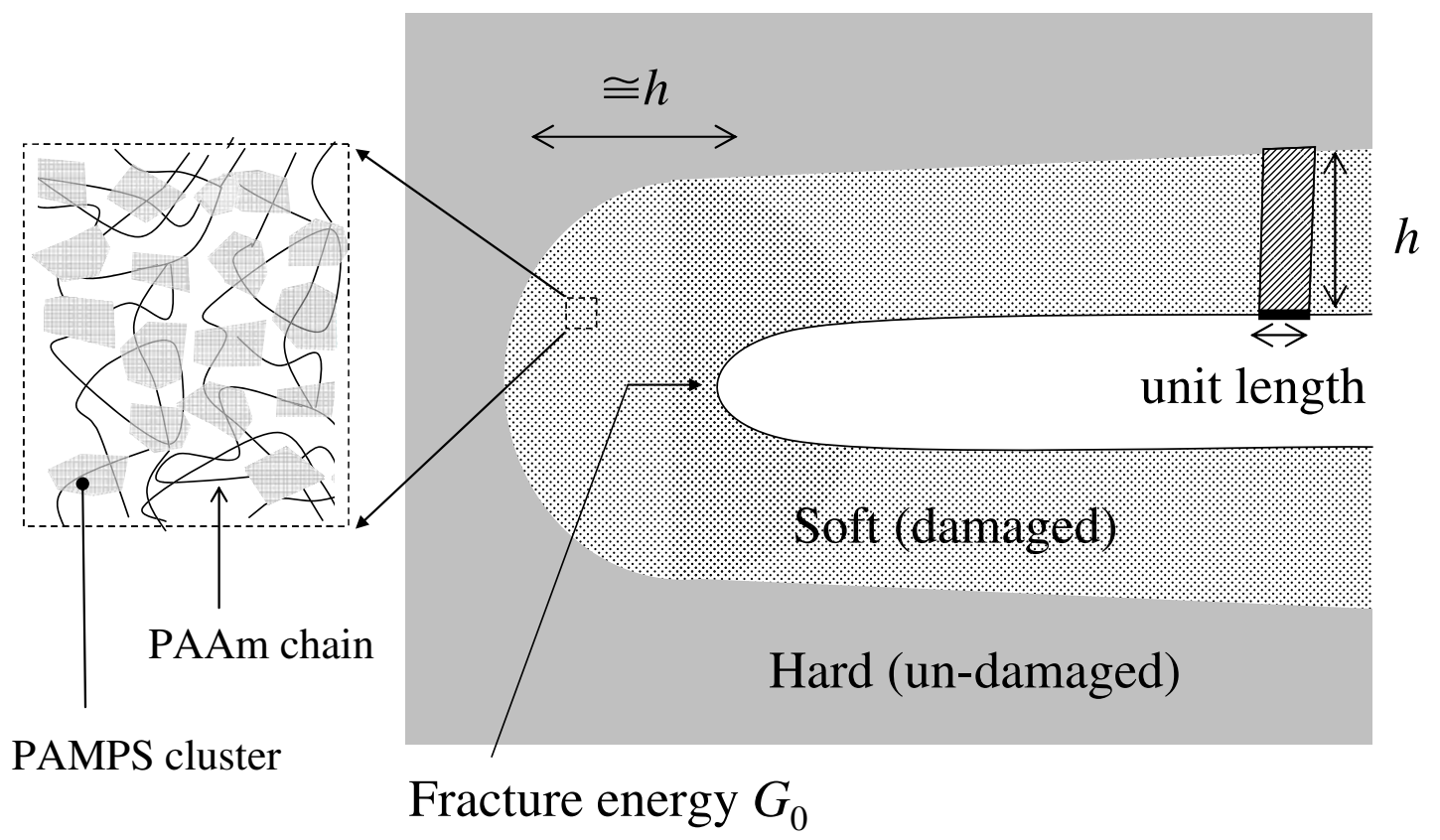

Fig. 1: The structure of crack assumed in the proposed model. The DN gel around the crack tip gets very soft due to the damage of the first PAMPS network. In the softened (damaged) zone, PAMPS clusters play a role of cross-linker of PAAm chains (the left illustration).

sliding mechanism is also inconsistent with the very weak dependence on $V$, because according to the mechanism, $G$ should be roughly proportional to $V[8]$. The bulk viscoelasticity effect cannot work well, because the bulk rheological property of the DN gels is dominated by the first network and shows no remarkable viscoelasticity.

A kind of yielding phenomena, which seems to be a clue to clarify the mechanics under high strength, has been found in DN gels made from more sparse first networks [10]; on tensile tests, narrowing zones ("necks") appear in the sample and grow up with further stretching. During the neck propagation, a plateau region appears in the loading curve. The plateau value of the tensile stress hardly depends on the stretching rate. After the neck propagation, the gels become fairly soft (but not sol), and can withstand large elongations (up to elongational strains around 20). It is certain that the first network breaks into small fragments during the necking deformation and the fragments play a role of cross-linker of the second PAAm chains (see fig. 1). The necking phenomenon can be regarded as a damage accumulation of the first network, and proposes a fascinating hypothesis for the anomalous toughness of the original tough DN gels [7]. That is, if the softened zone is formed in a mesoscale region around the crack tip (fig. 1), the crack propagation involves a large energy dissipation, resulting in the enhancement of the effective fracture energy. The situation is similar to crazing in the crack front of glassy polymers [11]. An advantage of the hypothesis is that it does not conflict with F5, i.e., the dissipation due to the yielding is expected to be insensitive to $V$, compared with viscous dissipations.

In this paper, following the line of the hypothesis, we propose a simple model to estimate the effect of the local damage on the observed fracture energy. The model assumes that in the strongly stretched region ahead of the crack tip, the gel first yields to transform into a very soft material with intrinsic fracture energy $G_{0}$, and then the crack tip passes through the softened zone. Using the energy balance concept of fracture mechanics, we obtain scaling level expressions of the size of the softened zone $h$ and of the effective fracture energy $G$, in terms of the yielding stress, the "bare fracture energy" $G_{0}$, and the typical strain and elastic constant in the softened zone. An order estimation of $G$ using the numerical data from the necking gel in [10] predicts that $G$ reaches several hundreds $\mathrm{J} / \mathrm{m}^{2}$. This is consistent with the experimental values of $G, \sim 400 \mathrm{~J} / \mathrm{m}^{2}[2]$.

Figure 1 depicts the structure of the crack. The damaged zone is created around the crack tip, and left on the both side of the fracture surfaces. We make the following assumptions: A1) The yielding occurs at a critical condition characterized by a threshold stress $\sigma_{\mathrm{c}}$, which corresponds to the critical tensile stress for the necking in the gels undergoing macroscopic necking. A2) There is a sharp boundary between the damaged and undamaged zones, and the size of the damaged zone can be characterized by only one spatial scale $h$ ( $h$ is the size 
(a)

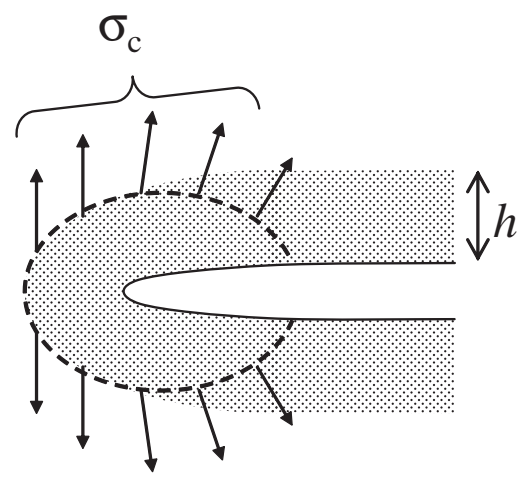

(b)

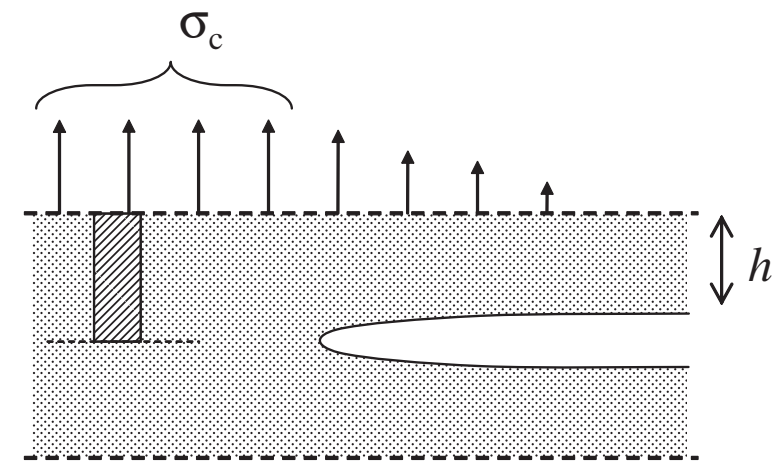

(c)

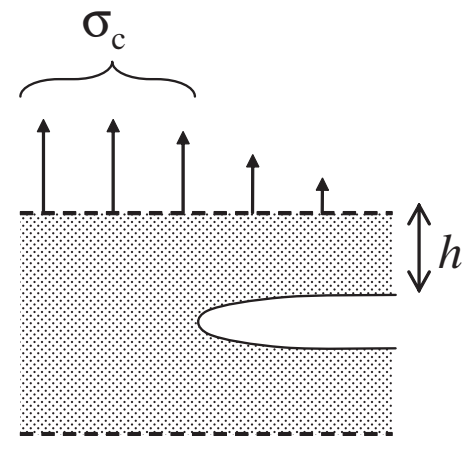

Fig. 2: (a) A close-up view of the damaged zone around the crack tip. The dashed curve shows the contour on which the principal tensile stress is given by $\sigma_{\mathrm{c}}$. The curve should include the "active" part of the boundary where the yielding occurs. (b) A virtual strip of the damaged material under pure shear test with the remote stress $\sigma_{\mathrm{c}}$, which has a similarity to the situation in (a). (c) Finite-length version of the pure shear test in (b).

in the reference (undeformed) state). A3) The damaged zone behaves as a very soft and purely elastic material with intrinsic fracture energy $G_{0}$.

We suppose a steady and quasi-static crack propagation, i.e., the boundary and the elastic field on both sides of the boundary uniformly shift with the crack propagation, and the process is not concerned with any dynamic (i.e., inertial and/or viscous) effects. Such process can be realized, for example, in very slow tearing tests.

Figure 2(a) is a close-up view around the crack tip. The critical condition for yielding (softening) is just satisfied on a part of the boundary ahead of the crack tip, and the yielding occurs there. The dashed curve schematically represent the contour on which the principal tensile stress is $\sigma_{\mathrm{c}}$. The contour should include the yielding boundary. On the other hand, the critical condition for the fracture is always satisfied at the crack tip. The size $h$ can be determined by the compatibility of the above two critical conditions.

The situation is similar to the so-called pure shear test shown in fig. 2(b), namely, the uniform expansion of an infinite strip including a half-infinite crack loaded with the remote stress $\sigma_{\mathrm{c}}$. The critical size (width) $h$ above which the crack can propagate at given $G_{0}$ and $\sigma_{\text {c }}$ can be determined by balancing the surface energy cost and the elastic energy release for a crack propagation at a unit length. The cost is $G_{0}$ (we assume the material has a unit length along the crack tip line, normal to the plane of fig. 2). The elastic energy release $g$ for this simple geometry is equal to the elastic energy stored in a uniformly stretched strip far away from the crack tip (hatched in fig. 2(b)) that has height $h$ and width 1 in the reference state. We have $g=h \times U\left(\sigma_{\mathrm{c}}\right)$, where $U\left(\sigma_{\mathrm{c}}\right)$ is the elastic energy density $\left(\mathrm{J} / \mathrm{m}^{3}\right)$ for the uniform stretching with critical stress $\sigma_{\mathrm{c}}$, and

$$
h=G_{0} / U\left(\sigma_{\mathrm{c}}\right)
$$

It should be noticed that eq. (1) is valid for large elastic deformations. Furthermore, eq. (1) is valid also for finitelength cases (fig. 2(c)) provided that the lateral size is several times as long as $h$. This is because the loading far away from the crack tip does not affect the stress field near the crack tip and because $h$ is the only characteristic scale to distinguish "far away" or not.

Equation (1) holds for the situation in fig. 2(a), provided we employ a suitable form of $U$ reflecting the complex boundary condition. (This is because eq. (1) is a consequence of the energy balance concept in fracture mechanics.) For the present purpose of order estimation, however, we can use the form of $U$ for the uniaxial stretching, because fig. 2(a) is equivalent to fig. 2(c) (and fig. 2(b)) on the scaling level. In this case, eq. (1) should be rewritten as $h \cong G_{0} / U\left(\sigma_{\mathrm{c}}\right)$, where the symbol "" represents that the dominant terms on both sides are equal, except for the numerical coefficients. The meaning of eq. (1) for our primary problem of fig. 2(a) is as follows: since the order of stresses on the boundary (and the corresponding elastic energy density) is limited by $\sigma_{\mathrm{c}}$, the damaged zone should largely grow to give enough elastic energy release to compensate large $G_{0}$.

Then, we explore the relation between $h$ and the effective fracture energy $G$, the form of $U\left(\sigma_{\mathrm{c}}\right)$ remaining unfixed. $G$ should be defined as the total dissipated energy to create a unit area of the fracture surface (we assume the material has a unit length along the crack tip line, normal to the plane of fig. 1). The dissipated energy consists of i) the base term $G_{0}$ and of ii) an added term 


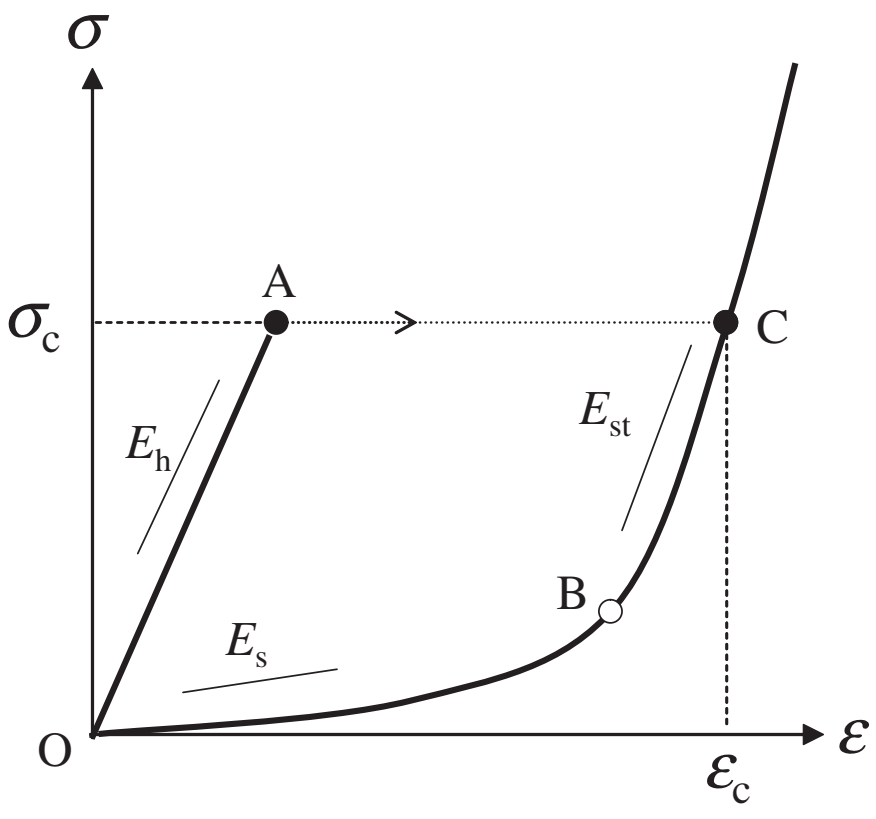

Fig. 3: Assumed stress-strain (S-S) relations for the undamaged and damaged zones. The state point irreversibly jumps from $\mathrm{A}$ to $\mathrm{C}$, corresponding to the damage. The S-S relation for the damaged material (OBC) is characterized by initial low modulus and stiffened modulus.

of irreversible work to cause the damaging (softening) in the strip $h \times 1$ (hatched in fig. 1). To estimate the order of the latter term, we ignore all complexities coming from the tensor nature of the problem, and consider a simplified problem of uniaxial stretching. Figure 3 shows the idealized loading curves. The stress-strain (S-S) relation of the undamaged sample follows the line OA with a high elastic modulus $E_{\mathrm{h}}$. At critical stress $\sigma_{\mathrm{c}}$, the state of the system jumps to $\mathrm{C}$, i.e., the softening occurs. After that, the S-S relation of the damaged material follows the curve OBC. The point B stands for the crossover region between the small deformation regime with low elastic modulus $E_{\mathrm{s}}$ and the large deformation regime with stiffened modulus $E_{\mathrm{st}}$. The area of the closed curve OACB is the irreversible work for the unit volume of the material, approximating to $\sigma_{\mathrm{c}} \times \varepsilon_{\mathrm{c}}$, where $\varepsilon_{\mathrm{c}}$ is the strain at the point $\mathrm{C}$. For the strip on the fracture surface, the irreversible work is $\sigma_{\mathrm{c}} \varepsilon_{\mathrm{c}} h$. Thus, we obtain an expression for the effective fracture energy $G$ :

$$
G=G_{0}+\sigma_{\mathrm{c}} \varepsilon_{\mathrm{c}} h=G_{0} \times\left(1+\frac{\sigma_{\mathrm{c}} \varepsilon_{\mathrm{c}}}{U\left(\sigma_{\mathrm{c}}\right)}\right) .
$$

It should be noticed that $G$ is given by a product of the bare fracture energy $G_{0}$ and a dimensionless enhancement factor.

To evaluate the size of the damaged zone $h$ and the effective fracture energy $G$, we need to know adequate values of $G_{0}, \sigma_{\mathrm{c}}, \varepsilon_{\mathrm{c}}$, and $U\left(\sigma_{\mathrm{c}}\right)$ for the tough DN gels. The bare fracture energy $G_{0}$ is estimated at around $10 \mathrm{~J} / \mathrm{m}^{2}$ [2]

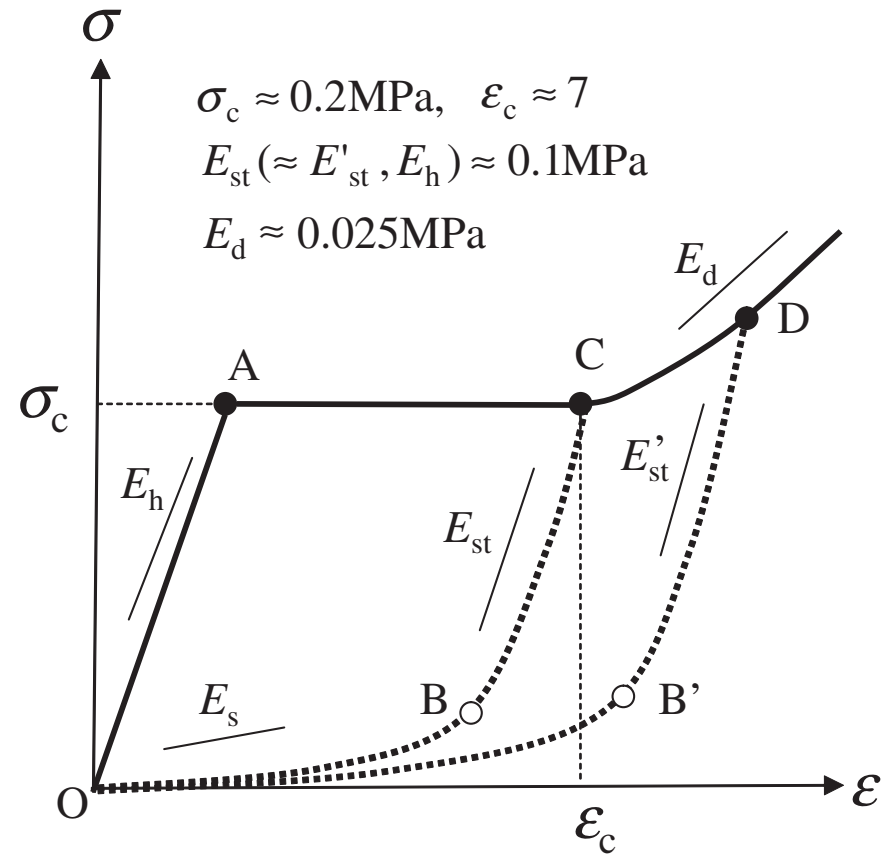

Fig. 4: Schematic representation of mechanical behavior of actual necking gels drawn based on the loading curves in figs. 1-3 in [10], and approximate values of the relevant quantities. The bold curve OACD represents the loading behavior of the virgin sample. Even above the point $\mathrm{C}$ (i.e., after the necking propagation) the damage accumulation gradually proceeds. The dotted curve OC (OD) shows the elastic stress-strain relation for the sample retracted from the point $\mathrm{C}(\mathrm{D})$.

on the basis that we can regard the softened zone as a permanent network of PAAm chains and can apply the Lake-Thomas mechanism [7]. (This value is reasonable, because it is a tenth of the typical value of fracture energy for rubbers, corresponding to the ratio of polymer contents of rubber, $100 \%$, and of DN gels, $15 \%$.) For $\sigma_{\mathrm{c}}, \varepsilon_{\mathrm{c}}$ and $U\left(\sigma_{\mathrm{c}}\right)$, there is no numerical data; thus we estimate the orders of the quantities with the experimental loading curve of a gel undergoing global (unlocalized) necking [10]. Based on figs. 1-3 in [10], fig. 4 schematically represents the loading behavior of the necking gel, and summarizes the approximate values of relevant quantities $\left(\sigma_{\mathrm{c}}\right.$ etc.). The curve OACD corresponds to the tensile loading for the virgin sample, and the plateau $\mathrm{AC}$ to the coexistence region of the necked and unnecked gels; $\sigma_{\mathrm{c}} \approx 0.2 \mathrm{MPa}$ and $\varepsilon_{\mathrm{c}} \approx 7$ as given in fig. 4. Accordingly we employ the order relations of $\sigma_{\mathrm{c}} \sim 10^{5} \mathrm{~Pa}$ and $\varepsilon_{\mathrm{c}} \sim 10$. (Hereafter, the symbol " $\sim$ " represents results of the order estimations based on the data from the necking gel.) When the stretched sample is retracted from the termination point of the coexistence region $\mathrm{C}$, it obeys the elastic $\mathrm{S}-\mathrm{S}$ relation of OBC. The S-S curve contains a stiffened region below $\mathrm{C}$ (see fig. 2 in [10] for the actual shape of the curve), and thus a reasonable choice for $U\left(\sigma_{\mathrm{c}}\right)$ is $U\left(\sigma_{\mathrm{c}}\right) \cong \sigma_{\mathrm{c}}^{2} / E_{\mathrm{st}}$ with $E_{\mathrm{st}} \approx 0.1 \mathrm{MPa}$. 
From eqs. (1), (2) and the order relations $G_{0} \sim 10 \mathrm{~J} / \mathrm{m}^{2}$, $\sigma_{\mathrm{c}} \sim E_{\mathrm{st}} \sim 10^{5} \mathrm{~Pa}$ and $\varepsilon_{\mathrm{c}} \sim 10$, we have

$$
\begin{gathered}
h \cong \frac{E_{\mathrm{st}} G_{0}}{\sigma_{\mathrm{c}}^{2}} \sim 100 \mu \mathrm{m}, \\
G \cong G_{0} \times\left(1+\frac{E_{\mathrm{st}}}{\sigma_{\mathrm{c}}} \varepsilon_{\mathrm{c}}\right) \cong G_{0} \times \frac{E_{\mathrm{st}}}{\sigma_{\mathrm{c}}} \varepsilon_{\mathrm{c}} \sim 100 \mathrm{~J} / \mathrm{m}^{2} .
\end{gathered}
$$

That is, the effective fracture energy $G$ could reach the order of several hundreds $\mathrm{J} / \mathrm{m}^{2}$. This is consistent with the measured value of $G$ of the $\mathrm{DN}$ gel.

Discussion. - We first clarify the physical meaning of the enhancement factor, $G / G_{0} \cong\left(E_{\mathrm{st}} / \sigma_{\mathrm{c}}\right) \varepsilon_{\mathrm{c}}$ in eq. (4). i) The inverse proportionality to $\sigma_{\mathrm{c}}$ is a consequence of two opposite effects of $\sigma_{\mathrm{c}}$ on $G$; one is enhancing the irreversible work $\sigma_{\mathrm{c}} \times \varepsilon_{\mathrm{c}}$, the other reducing $h \cong G_{0} E_{\mathrm{st}} / \sigma_{\mathrm{c}}^{2}$. The latter is stronger, resulting in the negative dependence of $G$ on $\sigma_{\mathrm{c}}$. ii) $\varepsilon_{\mathrm{c}}$ comes from the irreversible work for the damaging. The proportionality to $\varepsilon_{\mathrm{c}}$ is reasonable from the molecular point of view: the origin of the irreversible work is the breakage of chemical bonds of the first network during the softening deformation; large $\varepsilon_{\mathrm{c}}$ corresponds to persistent and multiple bond cuttings. iii) $E_{\text {st }}$ comes from $h$; if $E_{\text {st }}$ is large, the elastic energy density is small at given $G_{0}$ and $\sigma_{\mathrm{c}}$, leading to large $h$ and to large $G$.

Before concluding, it should be pointed out that for the necking gel in [10], the damage accumulation continues even after the neck propagation (above $\mathrm{C}$ ), and that the assumption A3 of the present model can be incorrect for the tough DN gels. As represented in fig. 4 (and as is clear in figs. 2 and 3 in [10]), i) the slope $E_{\mathrm{d}}$ of the loading curve after the neck propagation is fairly smaller than $E_{\text {st }}$; and ii) when the stretched sample is retracted from a point $\mathrm{D}$ in the post-necking stage, it shows an elastic S-S relation OD with a stiffened modulus $E_{\mathrm{st}}^{\prime}>E_{\mathrm{d}}$. These behaviors indicate that the S-S relation of the softened gel shifts depending on the maximum stretching suffered before, and that when the stretching of a gel exceeds the "personal highest" value, the slope of the S-S curve discontinuously decreases. Because of the additional damage accumulation following the drastic necking, the true irreversible work should be larger than $\sigma_{\mathrm{c}} \times \varepsilon_{\mathrm{c}}{ }^{1}$.
We believe, nevertheless, that the assumption A3 is useful as the first approximation and that eq. (4) is appropriate for the purpose of the order estimation of the effective fracture energy of the DN gels.

In conclusion, we proposed a simple phenomenological model that can qualitatively explain the anomalous high toughness of DN gels. The central idea is that the DN gel locally yields to become a softer material, and the energy dissipation by the yielding enhances the effective fracture energy. Microscopically, the yielding is supposed to be caused by damage accumulation and fragmentation of the brittle first network and inter-connection of the fragments by the second polymer chains.

$$
* * *
$$

The author thanks T. NAKAJIMA, Y. KAWAUChI, H. Furukawa and J. P. Gong for discussions and comments. He is grateful to the referees for the helpful comments.

\section{REFERENCES}

[1] Gong J. P., Katsuyama Y., Kurokawa T. and Osada Y., Adv. Mater., 15 (2003) 1155.

[2] Tanaka Y., Kuwabara R., Na Y.-H., Kurokawa T., Gong J. P. and Osada Y., J. Phys. Chem. B, 109 (2005) 11559.

[3] Greensmith H. W., J. Polym. Sci., 21 (1956) 75.

[4] Na Y.-H., Kurokawa T., Katsuyama Y., Tsukeshiba H., Gong J. P., Osada Y., Okabe S., Karino T. and ShiBAYAMA M., Macromolecules, 37 (2004) 5370.

[5] Tsukeshiba H., Huang M., Na Y.-H., Kurokawa T., Kuwabara R., Tanaka Y., Furukawa H., Osada Y. and Gong J. P., J. Phys. Chem. B, 109 (2005) 16304.

[6] Okumura K., Europhys. Lett., 67 (2004) 470.

[7] Lake G. J. and Thomas A. G., Proc. R. Soc. London, 300 (1967) 108.

[8] Baumberger T., Caroli C. and Martina D., Nat. Mater., 3 (2006) 522.

[9] DE Gennes P. G., Langmuir, 12 (1996) 4497.

[10] Na Y.-H., Tanaka Y., Kurokawa T., Furukawa H., Sumiyoshi T., Gong J. P. and Osada Y., Macromolecules, 39 (2006) 4641.

[11] Brown H. R., Macromolecules, 24 (1991) 2752.

\footnotetext{
${ }^{1}$ In the third paragraph in the page 4643 of [10], we made a misleading description about the (time independent) hysteresis loop in the loading curves of the necking DN gel, ignoring the additional damage.
} 